\title{
Lesions associated with mineral deposition in the lymph nodes and lungs of cattle: a case-control study of environmental health hazard
}

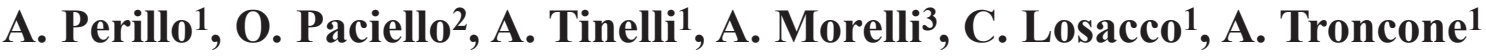 \\ ${ }^{1}$ Department of Animal Health and Welfare, Faculty of Veterinary Medicine, Bari, Italy \\ ${ }^{2}$ Department of Pathology and Animal Health, University of Naples Federico II, Naples, Italy \\ ${ }^{3}$ General practitioner Bari, Italy
}

\begin{abstract}
This report focuses on the state of health of the cattle raised in the district of Taranto - city of Italy rated as environmentally at risk. Representative samples of lungs, bronchial and mediastinal lymph nodes of cattle from district of Taranto's slaughterhouses were collected. After a macroscopic examination, samples with marked lesions were processed for light microscopy. Samples were also observed with polarized light microscopy, scanning electron microscopy and with microanalysis. The macroscopic examination revealed that 60 out of 183 samples showed marked lesions. Lung alterations were characterized by thickening of the alveolar septa and by the latter's modifying action on the alveolar spaces, foci of fibrosis and bronchopulmonary inflammation. For 51 out of the 60 samples observed, the histological examination confirmed the presence of pneumoconiosis and lymph nodal anthracosis. Energy-dispersive X-ray microanalysis of lung samples identified a wide range of elements including silicon, aluminium, titanium, iron, carbon and small amount of the other metals. In the lymph-nodes the same kind of metals with a different levels of distribution was observed. Our survey on cattle farmed in areas at high risk of pollution may be helpful to the estimation of the exposure risk for man to environmental contaminants and to the evaluation of the occurrence of the pathological manifestations as well.
\end{abstract}

Keywords: pulmonary fibrosis, lymph-node, environmental pollution, cattle, mineral.

\section{Introduction}

The presence of polluting substances in the atmosphere is a phenomenon largely related to urban and industrial areas. It is here that the emissions sources from heating plants, industrial activities and motorvehicle waste gases are mostly concentrated. Such substances can exert damaging effects to both human and environmental health, according to the contaminant's quantities being considered, the exposure time, and the harmful effects of the substance in question [1].

A decisive influence on the concentration of polluting-agents is performed by the weather conditions, which can either encourage or inhibit the spreading of contaminants in the atmosphere. Among the earth's atmosphere contaminants, sulphur dioxide, carbon

Correspondence: O. Paciello, Dept. of Pathology and Animal Health, University of Naples Federico II, Via Delpino, 1 - 80137, Naples, Italy; tel.: (+39) 0812536466, fax.: (+39) 0812536186, e-mail: paciello@unina.it dioxide, carbon monoxide, nitrogen oxides, and gaseous hydrocarbons are to be enumerated. With respect to gaseous hydrocarbons, which are emitted after combustion as a particulate, the term here employed is generically referred to substances present in the air in the form of solid or liquid particles. In addition to the aforesaid substances, several heavy metals, such as lead, mercury and zinc are normally present. Although in modest amounts, in the air, in the water and in the soil. When in high concentration, such metals do embody threat to the ecosystems. The dangerousness of heavy metals is due both to their nonbiodegradability and to the possibility of their accumulation effects within living organisms [1].

The respiratory system is the main target of many chemicals compounds' toxic effect. In consideration of its anatomical location, and of the function its exerts, the respiratory system is constantly in contact with noxious agents present in the atmosphere. It is also well known that most inhaled particles can build up in the lung's tributary lymph nodes. Inhaling is the main way through which target organs, lungs and lymph 
nodes chiefly, come into contact with the contaminants dispersed in the air $[2,3]$. Absorption of toxic substances depends on several factors, first of all the physical, chemical, anatomical and functional properties of the cardio-respiratory system. The absorption mechanism can differ whether it interests a gas or a liquid substance. Nonetheless, in general the absorptioncapacity in the lung increases as solubility in water increases. Highly hydro soluble gases, such as, sulphur dioxide $\left(\mathrm{SO}_{2}\right)$, do not usually go beyond the nasopharynx. On the contrary, poorly hydro soluble gases, such as nitrogen dioxide $\left(\mathrm{NO}_{2}\right)$, or the hydro soluble gases adsorbed by the particulate or in the form of aerosol, can reach the bronchial tree's peripheral areas thus inducing a toxic reaction. High concentrations of such substances can provoke disorders and diseases [4-7]. Among the most representative morphological alterations which interest the respiratory system, fibrosis, emphysema and neoplasias are to be mentioned [4-7]. Contaminants of a factory origin which can most frequently cause such pathologies are: asbestos, cadmium, arsenic, sulphur dioxide, ozone, formaldehyde.

Domestic as well as wild animals are exposed, just like man, to contaminants present in the air, in the soil, in the water and in food. They can suffer from the potential acute and chronic effects of such exposure. Animal population exposed to contaminants in the environment are referred to as "animal sentinel systems" (ASS) [8,9]. These are designed to the identification or the monitoring of a variety of environmental contaminants dangerous to the human, animal and ecosystem health. A sentinel animal is defines like "one which points out or directs attention to something", and more specifically, sentinel animals are defines like "a group of animals whose presence acts as a sign of particular environmental conditions" [8,9].

The present study focused on cattle farmed in the district of Taranto, a city rated as environmentally at risk. The aim of our research was to assess the effects of the environmental pollution in lungs and tributary lymph nodes of cattle.

\section{Materials and methods}

183 samples from lungs and bronchial as well as mediastinal lymph nodes of cattle from district of Taranto's slaughterhouses were collected from September 2004 through November 2005. The animals were end-of-career milk cows, Bruna, Italian Frisona, Podolica and cross breeds. By consulting the daily slaughter - listtracing animals' serial number, age and company code - it was possible to infer that all animal were from pollutant districts.

After a careful macroscopic examination, samples with marked lesions were processed for light microscopy. Lung and bronchial lymph node samples were fixed in buffered formalin $10 \%$ and processed for paraffin embedding. Histological diagnosis was assessed on 6-nm-thick hematoxylin-eosin (HE) and Van-Giesonstained sections. In addition, some sections were stained with Ziehl-Neelsen method and with Fite's new fuchsine formaldehyde method for the acid-fast bacilli.
For scanning electron microscopy (SEM), lung and bronchial lymph-node samples were fixed in paraformaldehyde $4 \%$ in phosphate buffer, dehydrated in a graded ethanol series and critical point dried. Specimens were mounted on aluminium stubs and coated with a layer of gold in a polaron E 5400 sputter-coater and examined with a Cambridge Stereoscan 240 scanning electron microscope. Some samples were mounted on carbon stubs and coasted with a layer of carbon and analysed by means of an AN 10/25 S Link X-ray analytical system with an energy dispersive spectrometer (EDS).

\section{Results}

Lungs and lymph-nodes with pathologic changes associated to some infectious agent (other than small amounts of terminal pneumonia) were excluded from these studies. The macroscopic examination revealed that 60 out of 183 samples showed noticeable lesions related to air pollution.

Lung alterations were marked by thickening of the alveolar septa and by the latter are modifying actions on the alveolar spaces, initial foci of fibrosis and bronchopulmonary inflammation (Fig. 1-2). In some cases, the presence of small, barely visible blackish dots, identified as carbon particles within macrophages, would stood out in the subpleuric interstice principally, giving the lung a blackish colour (Fig. 1). Microscopically the medullary sinus macrophages had small need-like intracellular crystals and large stranslucent estracellular crystals observed with the polarized light microscopy (Fig. 3 and 4). In the subpleuric interstice, carbuncular particles present in macrophages' cytoplasm were accountable for a responsiveness of a fibrous kind, with a tendency towards interstice and alveolus invasion. Further histological investigation evidenced, moreover, a peribronchial fibrosis as well as lymph-plasmocyte and granulocyte infiltrates (Figs 5 and 6 ).

The accumulation-effect of given contaminants changes in a linear way as age increases, although lesions in animals no older than 4 or 5 months from a distant zone from the most-at-risk industrial pole were detected.

A blackish, diffused pigmentation marked the lung lymph nodes' surface once cut (Fig. 2). Carbon-dust accumulation in many samples would look as an organization of minute, dark spots arranged as a crown along the edge between cortex and medulla. Involved lymph nodes would appear normal or increased in volume, with a reactive hyperplasia area of variable entity (Fig. 2). Under both macroscopic and microscopic examination, a distinction between follicular hyperplasia phenomena, circulatory disorders, exudation imbibition and fibrosis proliferation was practicable.

The histological examination revealed that lymph node displayed a severe lymphoid depletion, an intense connective reaction which could alter the organ architecture completely. Sparse or aggregated 

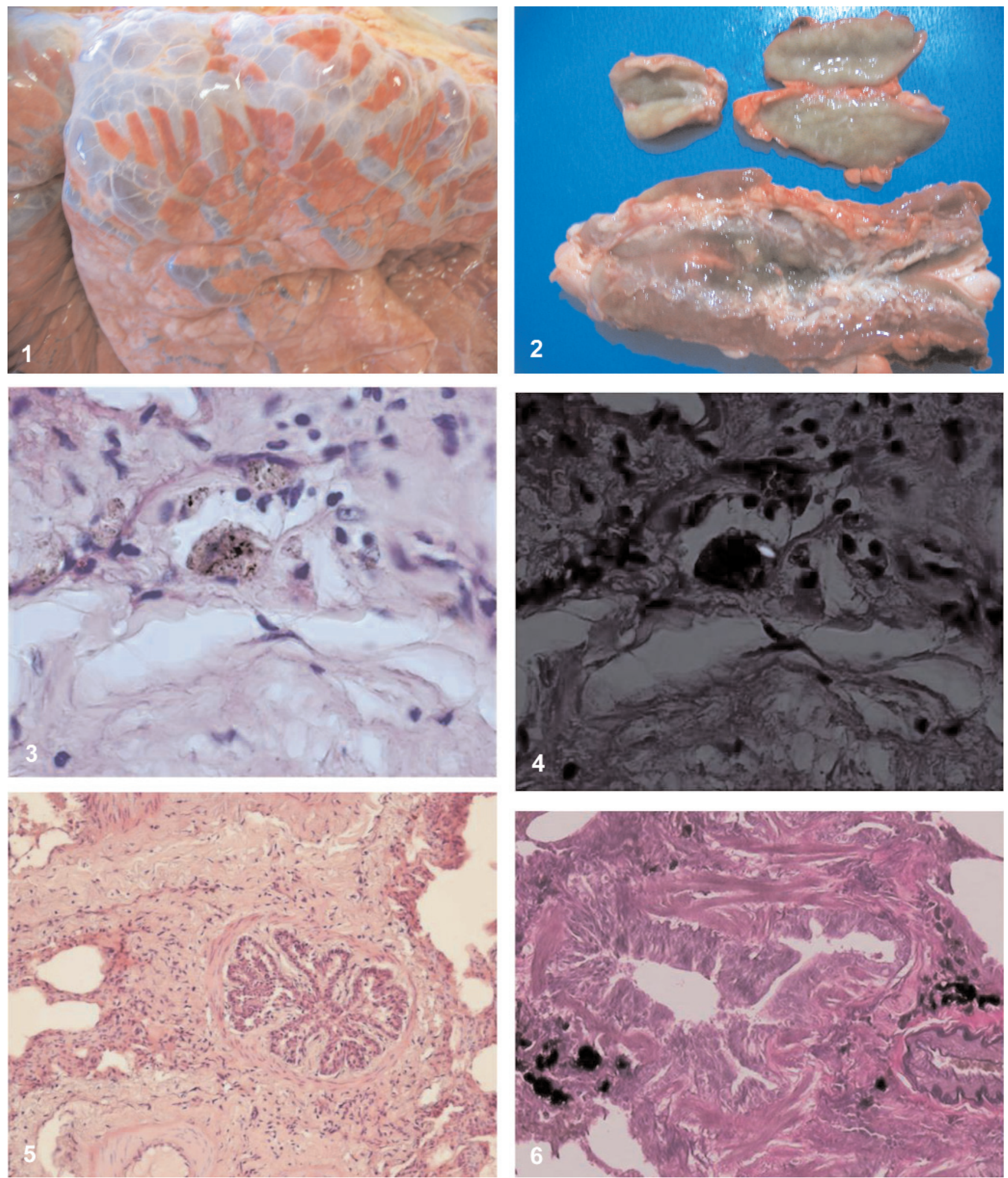

Fig. 1. Cattle, lung: pleural thickness and severe interstitial emphysema. Fig. 2. Cattle, lymph node: Mineral associated lymphadenopathy with follicular hyperplasia and little dots of blackish pigments in the parenchyma. Fig. 3. Cattle, lung: Presence of carboniotic exsogenous particles within macrophages $(\mathrm{H}+\mathrm{E}$ original magnification $\times 40)$. Fig. 4. Cattle, lung: macrophage with a large translucent intracellular crystals $(H+E$, polarized light. original magnification $\times 40)$. Fig. 5. Cattle, lung: Severe peribronchial fibrosis associated with muscular hypertrophy and broncospasm (E.E. original magnification $\times 20$ ). Fig. 6. Cattle, lung: Marked proliferation of peribronchial connective tissue associated with the presence of macrophages full of carbon particles (Van-Gieson original magnification $\times 10$ ). 

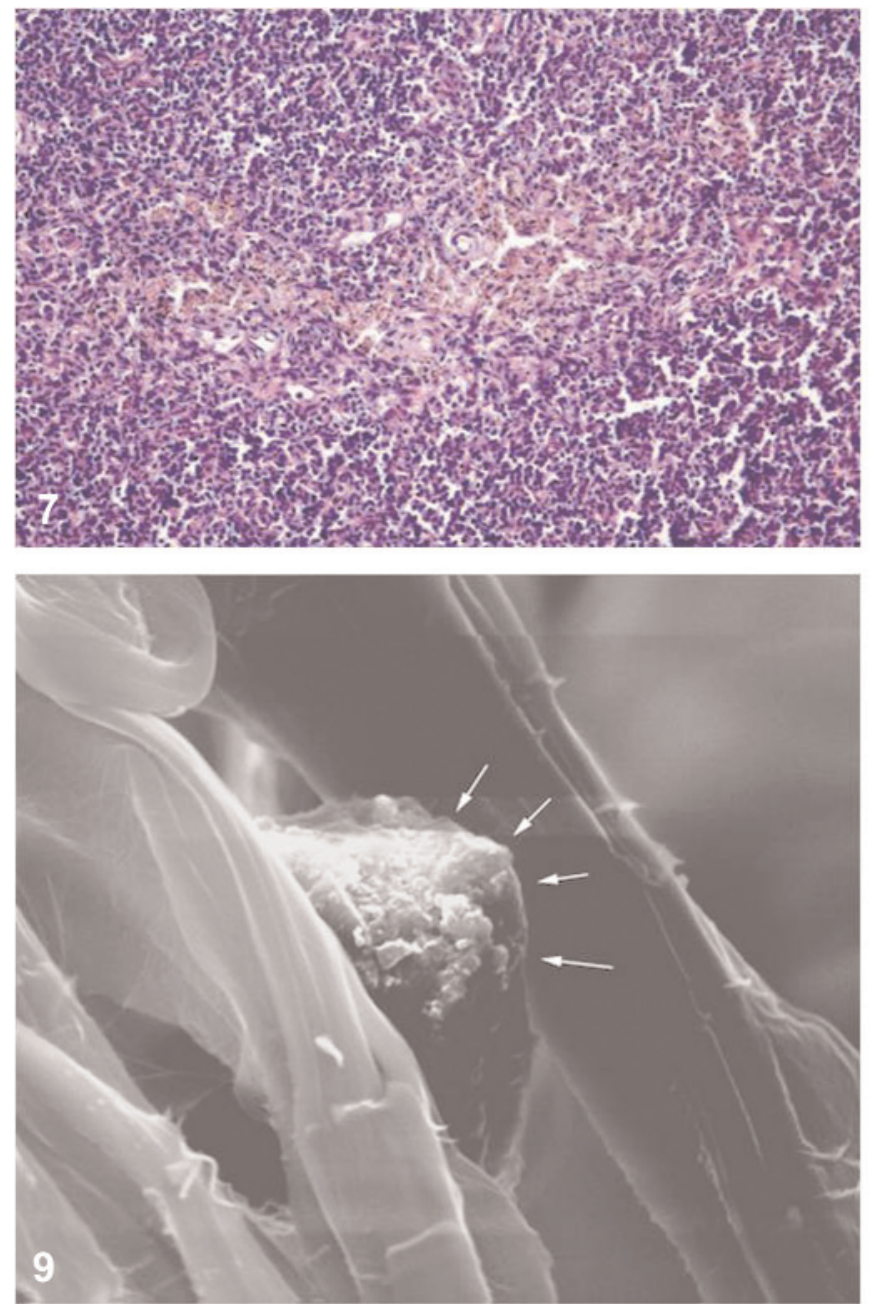

macrophages were present and rich in anthracotic and silicotic pigment (Fig. 7) along the edge between cortex and medulla, also revealed with polarized light microscopy (Fig. 8), in addition to a quantity of accumulated pigment in pseudo-cavities, together with haemorrhagic infiltrations.

For 51 out of the 60 samples observed, the histological examination confirmed the macroscopic diagnosis, i.e. the presence of pneumoconiosis and lymph nodal anthracosis. For the remaining part, the presence of pigmentation of other nature was found. The massive accumulation of pigments at the lung level, whether associated to lesions in the satellite lymph nodes or not, underlines an accumulation-effect of major entity and duration, contrasted by no efficient phagocytic response. Such is the typical clinical picture observable in older animals with general signs of wasting of the body and generalized lymph nodal sclerosis phenomena.

Particles deposition in the lung had different shapes, some of them were plate-like particles and other compact particles seated in the alveolar space (Fig. 9).

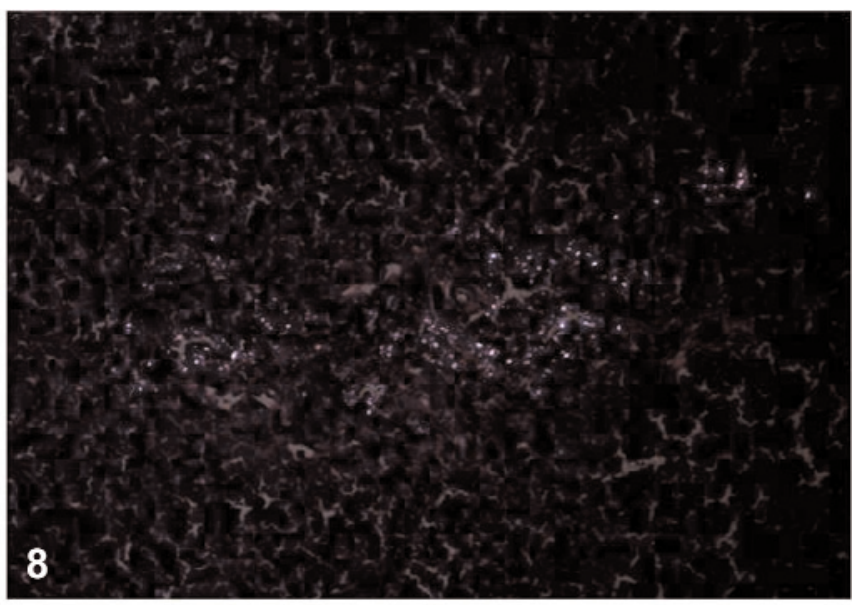

Fig. 7. Cattle, lymph node: large numbers of macrophages full of pigments in the medulla cortex (E.E. original magnification $\times 20$ ). Fig. 8. Cattle, lymph node: medulla cortex aggregates of macrophages with cytoplasmic carbon particles and translucent needlelike crystals $(\mathrm{H}+\mathrm{E}$, polarized light, original magnification $\times 20)$. Fig. 9. Cattle, lung: compact silicon particles attached on the alveolar wall (SEM, original magnification $\times 3400$ ).

Energy-dispersive X-ray microanalysis of some selected lung samples showed the material to contain carbon, aluminium, silicon, titanium, iron and small amount of the other metals (Fig. 10). In the lymphnodes we observed the same kind of metals with different levels of distribution (Fig. 11).

\section{Discussion}

The increasing in environmental pollution is responsible for interstitial chronic pnemopathies [10-12]. Normally, inhaled air dusts greater than $5 \mu \mathrm{m}$ are eliminated by the mucociliary transport. On the contrary, air particles little than $5 \mu \mathrm{m}$, can reach the deep where thy can induce macrophages to release chemical inflammatory substances which stimulate fibroblastic proliferation. Observed structural aspects and alterations amount to no apparent expression of assured severity and remain nearly always limited and localized. Nevertheless, they prove particularly significant and provide further evidence of a pulmonary and lymph nodal tissue reactive response to extraneous substances [13]. 

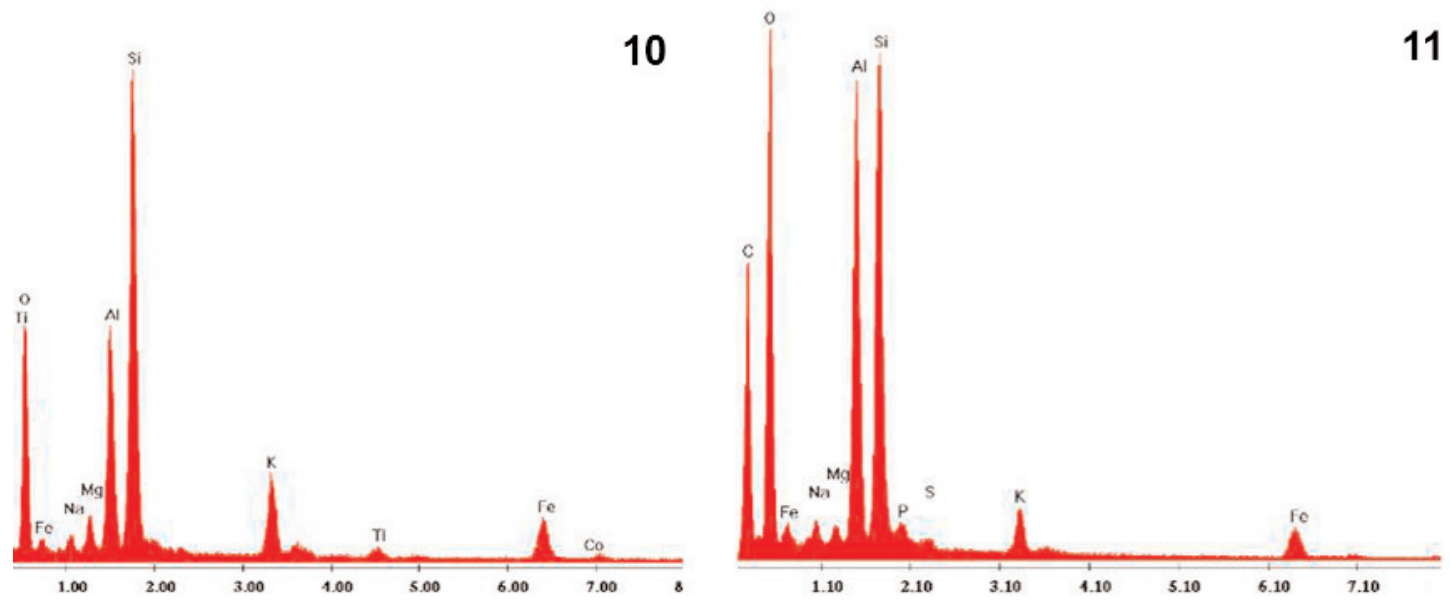

Fig. 10. Electron microprobe analysis of dust from representative lungs samples. The spectra demonstrate the heterogeneous character of the crystals. Fig. 11. Electron microprobe analysis of dust from representative lymph nodes with mineral associated lymphadenopathy. The spectra demonstrate a different composition and amount of the character of the crystals compared with lungs results.

The most remarkable aspect of our observations is fibrotic reactivity at peribronchial, perivascular, alveolo-capillary, and pleural levels.

It is well known that fibrotic process is central to tissue repair. When exceeding - if unchecked - the process can nonetheless alter organ's structure and compromise its physiological functions [14,15]. Pulmonary fibrosis is the final stereotypical pathologic event resulting from insults caused by various agents including infective agents, pharmaceuticals, chemicals, organic as well as inorganic dusts, gases, fumes, and vapours. Regrettably, the mechanism in charge of pulmonary fibrosis control is still to be completely clarified.

Experimental studies carried out on rats exposed to asbestos inhalation have shown how alveolar macrophages flock in great numbers while local macrophages take action and produce fibroblast growth factors (FGF) [5] exactly like mastocites produce b-FGF, cytokines playing key role in silicosis development [15].

In present study, the close association between connective tissue proliferation on the one hand, and macrophages activated by the presence of extraneous particulate material on the others, confirms both the central role being played by macrophages in fibrogenetic process modulation, and the importance of airdispersed dusts in chronic pneumopathies ascertaining.

Examined fibrotic lesions are always of modest entity and never emerge with the typical pneumoconiotic lesions. This may possibly be explained on account of either comparatively low percentage of dusts found - mainly referring to $\mathrm{Si}$, the sclerogenetic properties of which are well known in human medicine - or by the 'immunitary tolerance' mechanism. We can assume that if these animals have been exposed to dusts in environment since birth - that is to say prior to their immunitary maturity - they were able to develop some 'habit' to dust itself by means of the same mechanism enabling whichever organism to regard extraneous particles they come into contact with during their immunitary immaturity (i.e. when antibody production has not taken place yet) as 'self'.

Results obtained through the survey we carried out on animals living in well defined areas within the district of Taranto and exposed to the environmental contaminants action, allow us to make a few remarks.

The toxic effect of a contaminant depends on a number of factors, such as the toxic type, its chemical and physical properties, the animal's exposure quantity, duration and condition, the subject's immune - response capacity and the possible presence of any bio-protection mechanism. In the organs in questions, alterations imputable to the noxious action of substances present in the environment were found. Our survey on cattle farmed in areas at high risk of pollution may be helpful to the estimation of the exposure risk for man to given environmental contaminants and to the evaluation of the occurrence of the pathological manifestations as well. Statistical data have shown an increase, over the last decades, of the respiratory pathologies in man-young subjects included-ranging from the slightest (asthma, bronchitis) to the gravest ones (pulmonary carcinomas), which are chiefly observable among inhabitants of large metropolises.

Results recorded by us have turned out to be analogous. Even the youngest animals (aged 4 months) would display pulmonary lesions of different intensity, responsible for severe respiratory deficiency. Monitoring such animals has proved to be a most appropriate choice, in consideration of the fact that 
cattle can well represent successful indicators of environmental health hazards in a habitat shared with man as well.

Our study enables us to conclude that domestic animals exemplify a powerful model for research on environmental pathologies

\section{References}

[ 1] Costa DL, Dreher KL. Bioavailable transition metals in particulate matter mediate cardiopulmonary injury in healthy and compromised animal models. Environ Health Perspect. 1997; 105(Suppl 5):1053-60.

[2] Minister, Public Works and Government services: National Ambient Air Quality Objectives for Particulate Matter. Canadian Environmental Protection Act, 1998.

[3] Robbins. Pathologic Basis of disease. Sixth Edition: cotran, Kumar, Collins by W.B. Saunders Company Philadephia Pennsylvania - USA (1999).

[4] Day MJ, Pearson GR, Lucke VM, Lane SJ, Sparks RS. Lesions associated with mineral deposition in the lymph node and lung of the dog, Vet Path. 1996;33(1):29-42.

[5] Lemaire I et al. Alveolar macrophage stimulation of lung fibroblast growth in asbestos-induced pulmonary fibrosis. $\mathrm{Am}$ J Path. 1986, pp 122- 205.

[ 6] Driscoll KE, Hassenbein DG, Carter J, Poynter J, Asquith TN, Grant RA, Whitten J, Purdon MP, Takigiku R. Macrophage inflammatory proteins 1 and 2: expression by rat alveolar macrophages, fibroblasts, and epithelial cells and in rat lung after mineral dust exposure. Am J Respir Cell Mol Biol. 1993; $8(3): 311-8$

[ 7] Ho JC, Lam WK, Ooi GC, Wong MP, Lam JC, Ip MS, Tsang KW. Lymphoepithelioma-like carcinoma of the lung in a patient with silicosis. Eur Respir J. 2003;22(2):383-6.

[ 8] Backer LC, Grindem CB, Corbett WT, Cullins L, Hunter JL. Pet dogs as sentinels for environmental contamination, Sci Tot Environm. 2001;274:161-169.

[ 9] O'Brien DJ, Kaneene JB, Poppenga RH. The use of mammals as sentinels for human exposure to toxic contaminants in the environment. Environ Health Perspect. 1993;99:351-68.

[10] Bates DV. Health indices of the adverse effects of air pollution: the question of coherence. Environ Res. 1992;59:336-349.

[11] Pope III CA, Dockery DW, Schwartz J. Review of epidemiological evidence of health effects of particulate air pollution. Inhal Toxicol. 1995; 7:1-18.

[12] Plopper CG, Buckpitt AR, Evans MJ, Van Winkle LS, Fanucchi MV, Smiley-Jewell SM, Lakritz J, West J, Lawson G, Paige R, Larson SD, Schelegle ES, Joad JP, Pinkerton KE, Gershwin LJ, Miller LA, Wu R, Hyde DM. Asthma and other Lung Diseases Associated with Environmental Toxicants. In: 55th Annual Meeting of the American College of Veterinary Pathologists (ACVP) Meeting of the American Society of Clinical Pathology (ASVCP), ACVP and ASVCP (Eds.).

[13] Venkataraman C, Kao AS. Comparison of particle lung doses from the fine and coarse fractions of Urban PM-10 Aerosols. Inhal Toxicol. 1999;11:151-169.

[14] Inoue Y, King TE Jr, Tinkle SS, Dockstader K and Newman LS. Human mast cell basic fibroblast growth factor in pulmonary fibrotic disorders. Am J Path. 1996;149(6):2037-54.

[15] Hamada H, Vallyathan H, Cool CD, Barker E, Inoue Y, Newman LS. Mast cell basic fibroblast growth factor in silicosis. Am J Resp Crit Care Med. 2000;161(6):2026-34.

Submitted: 26 October, 2008 Accepted after reviews: 28 August, 2009 